EPJ Web of Conferences 112, 01021 (2016)

DOI: 10.1051/epjconf/20161120102 1

C) Owned by the authors, published by EDP Sciences, 2016

\title{
Non-dipolar gauge links for transverse-momentum-dependent pion wave functions
}

\author{
Yu-Ming Wang ${ }^{1, a}$ \\ ${ }^{1}$ Fakultät für Physik, Universität Wien, Boltzmanngasse 5, 1090 Vienna, Austria
}

\begin{abstract}
I discuss the factorization-compatible definitions of transverse-momentumdependent (TMD) pion wave functions which are fundamental theory inputs entering QCD factorization formulae for many hard exclusive processes. I will first demonstrate that the soft subtraction factor introduced to remove both rapidity and pinch singularities can be greatly reduced by making the maximal use of the freedom to construct the Wilson-line paths when defining the TMD wave functions. I will then turn to show that the newly proposed TMD definition with non-dipolar Wilson lines is equivalent to the one with dipolar gauge links and with a complicated soft function, to all orders of the perturbative expansion in the strong coupling, as far as the infrared behavior is concerned.
\end{abstract}

\section{Introduction}

Hard exclusive processes have significantly sharpened our understanding of the strong interaction dynamics involved in the hadronic amplitudes, due to the establishment of various QCD factorization theorems which allow for separation of the hard scattering contributions and the (universal) longdistance QCD effects. Albeit the fact that factorization in a non-abelian gauge theory like QCD is by no means a trivial issue, collinear factorization for a number of interesting hard reactions, at leading power in $1 / Q$ where $Q$ is the typical hard scale for a given process, can be established with rigorous power counting schemes and standard assumptions. By contrast, transverse-momentum-dependent (TMD) factorization for hard exclusive processes based upon the theory of on-shell Sudakov form factor [1], with the original aim of avoiding the rapidity divergences in collinear factorization for the pion electromagnetic form factor at sub-leading power [2], turns out to be far more sophisticated conceptually due to the absence of a definite power counting scheme and to the emergence of lightcone singularities in the infrared subtraction, and this can be already partially recognized from the troubled history of TMD wave functions [3-14].

The primary difficulty of defining TMD wave functions lies in eliminating the rapidity divergence involved in the infrared subtraction, due to the TMD hard scattering kernels, without introducing additional pinch singularities. It is the subjective of this presentation to overview the current status of TMD factorization for hard exclusive processes, focusing on the comparison between collinear and TMD pion wave functions and the regularization of unwanted rapidity and pinch divergences. I will consider the simplest hard exclusive process $\gamma^{*} \rightarrow \pi \gamma$ (neglecting the involved sub-leading contributions [15] for the moment) through the discussion in order not to bother about the complication due

\footnotetext{
a e-mail: yu-ming.wang@univie.ac.at
} 
to the Glauber/Coulomb gluon exchange [16] which are relevant to the construction of factorization theorems for more complicated processes, e.g., the pion electromagnetic form factor. It is certainly interesting to generalize the discussion presented here to understand the factorization properties of those processes taking into account the contribution from the Glauber gluons, which is also crucial to construct the Wilson line structures of TMD wave functions. In what follows, I will first explain the origin of light-cone singularities in the infrared subtraction determined by the convolution of TMD wave functions and the hard functions, and present new definitions of the TMD pion wave functions with dipolar and non-dipolar Wilson lines in section 2. Demonstration of the equivalence of different TMD definitions is then discussed with the aid of rapidity evolution equations in section 3 .

\section{Factorization-compatible TMD wave functions}

To facilitate the comparison between the collinear and TMD pion wave functions, I will begin with the collinear factorization formula for the pion-photon transition form factor [3]

$$
F_{\pi}\left(Q^{2}\right)=\frac{\sqrt{2} f_{\pi}}{3} \int_{0}^{1} d x H\left(x, Q^{2}, \mu\right) \phi_{\pi}(x, \mu),
$$

where the pion light-cone distribution amplitude $(\operatorname{LCDA}) \phi_{\pi}(x, \mu)$ is given by

$$
\left\langle 0\left|\bar{q}(0) W_{n_{-}}\left(0, t n_{-}\right) n_{-} \gamma_{5} q\left(t n_{-}\right)\right| \pi^{+}(p)\right\rangle=i f_{\pi} p_{+} \int_{0}^{1} d x e^{-i x t p_{+}} \phi_{\pi}(x, \mu),
$$

with the collinear gauge link

$$
W_{n_{-}}\left(0, t n_{-}\right)=P \exp \left[i g_{s} \int_{0}^{t} d \lambda T^{a} n_{-} \cdot A^{a}\left(\lambda n_{-}\right)\right] .
$$

The hard function $H\left(x, Q^{2}, \mu\right)$ determined from the perturbative QCD matching is known to the nextto-next-to-leading order in the strong coupling [17]. Taking into account the Sudakov form factor to suppress the soft gluon contribution [2], the TMD factorization formula for the $\gamma^{*} \rightarrow \pi \gamma$ form factor can be written as

$$
F_{\pi}\left(Q^{2}\right)=\frac{\sqrt{2} f_{\pi}}{3} \int_{0}^{1} d x \int d^{2} \vec{b} H\left(x, \vec{b}, Q^{2}, \mu\right) \phi_{\pi}(x, \vec{b}, \mu) e^{-S(x, \vec{b}, Q)},
$$

where $S(x, \vec{b}, Q)$ is the perturbative Sudakov form factor and the non-perturbative Sudakov evolution is left out due to the strong model dependence. Naively, one may expect the following definition of the TMD pion wave function in momentum space

$$
\begin{aligned}
\phi_{\pi}^{\text {naive }}\left(x, \vec{k}_{T}, \mu\right) \stackrel{?}{=} & \int \frac{d z_{-}}{2 \pi} \int \frac{d^{2} z_{T}}{(2 \pi)^{2}} e^{i\left(x p_{+} z_{-}-\vec{k}_{T} \cdot \vec{z}_{T}\right)} \\
& \times\langle 0| \bar{q}(0) W_{n_{-}}^{\dagger}(+\infty, 0) \not h_{-} \gamma_{5}\left[\text { tr. link] } W_{n_{-}}(+\infty, z) q(z)\left|\pi^{+}(p)\right\rangle,\right.
\end{aligned}
$$

with the coordinate $z=\left(0, z_{-}, \vec{z}_{T}\right)$, which can be constructed from a straightforward extension of (2). Unfortunately, the above-mentioned definition yields the light-cone divergence in the infrared subtraction, which can be readily understood from the one-loop correction to the quark-Wilson-line diagram

$$
\phi_{\pi}^{(1)} \otimes H^{(0)} \propto \int[d l] \frac{1}{\left.\left[(k+l)^{2}+i 0\right)\right]\left[l_{+}+i 0\right]\left[l^{2}+i 0\right]}\left[H^{(0)}\left(x+l_{+} / p_{+}, \vec{k}_{T}+\vec{l}_{T}\right)-H^{(0)}\left(x, \vec{k}_{T}\right)\right] .
$$


In contrast to the collinear factorization, this loop integral develops an endpoint singularity from the $1 / l_{+}$singularity in the Eikonal propagator, due to the non-cancellation of two hard functions at $l_{+}=0$. Two different definitions were proposed [4] to regularize such rapidity divergence either by rotating the gauge links off the light cone or by introducing an additional subtraction factor

$$
\begin{aligned}
\phi_{\pi}^{C 1}\left(x, \vec{k}_{T}, y_{u}, \mu\right) \stackrel{?}{=} & \int \frac{d z_{-}}{2 \pi} \int \frac{d^{2} z_{T}}{(2 \pi)^{2}} e^{i\left(x p_{+} z_{-}-\vec{k}_{T} \cdot \vec{z}_{T}\right)} \\
& \times\langle 0| \bar{q}(0) W_{u}^{\dagger}(+\infty, 0) \not h_{-} \gamma_{5} \text { [tr. link] } W_{u}(+\infty, z) q(z)\left|\pi^{+}(p)\right\rangle, \\
\phi_{\pi}^{C 2}\left(x, \vec{k}_{T}, y_{u}, \mu\right) \stackrel{?}{=} & \int \frac{d z_{-}}{2 \pi} \int \frac{d^{2} z_{T}}{(2 \pi)^{2}} e^{i\left(x p_{+} z_{-}-\vec{k}_{T} \cdot \vec{z}_{T}\right)} \\
& \times \frac{\langle 0| \bar{q}(0) W_{n_{-}}^{\dagger}(+\infty, 0) \not n_{-} \gamma_{5}\left[\operatorname{tr} . \text { link] } W_{n_{-}}(+\infty, z) q(z)\left|\pi^{+}(p)\right\rangle\right.}{\langle 0| W_{n_{-}}^{\dagger}(+\infty, 0) W_{u}(+\infty, 0)\left[\operatorname{tr} . \text { link] } W_{n_{-}}(+\infty, z) W_{u}^{\dagger}(+\infty, z)|0\rangle\right.},
\end{aligned}
$$

where the gauge vector $u=\left(u_{+}, u_{-}, \overrightarrow{0}_{T}\right)$ is away from the light cone.

Some years later, Bacchetta et al [18] found that the TMD definitions like (7) and (8) will induce the pinch singularity for a TMD parton density with space-like Wilson lines and hence they cannot be used to construct TMD factorization formulae for the hard inclusive reactions in practice. Even worse, the pinch singularity appears in TMD wave functions for any off-light-cone gauge vector, because the radiative gluon is not necessary to be on the mass shell [19]. To develop a better understanding of the resulting pinch singularity, one can compute the Wilson line self-energy graph explicitly

$$
\phi_{\pi} \propto \int[d l] \frac{u^{2}}{[l+i 0)][u \cdot l+i 0][u \cdot l-i 0]} \delta\left(x^{\prime}-x+l_{+} / p_{+}\right) \delta^{(2)}\left(\vec{k}_{T}^{\prime}-\vec{k}_{T}+\vec{l}_{T}\right),
$$

which yields the linear divergence in the length of the Wilson lines in coordinate space. One then concludes that off-light-cone dipolar Wilson lines regularize the rapidity divergence at the price of introducing an unwanted pinch singularity.

The problem of defining factorization-compatible TMD definitions was resolved only recently by Collins [16] in the context of parton densities. Generalizing his proposal to the TMD wave functions one obtains

$$
\begin{aligned}
\phi_{\pi}^{C}\left(x, \vec{k}_{T}, y_{2}, \mu\right)= & \lim _{\substack{y_{1} \rightarrow+\infty \\
y_{u} \rightarrow-\infty}} \int \frac{d z_{-}}{2 \pi} \int \frac{d^{2} z_{T}}{(2 \pi)^{2}} e^{i\left(x p_{+} z_{-}-\vec{k}_{T} \cdot \vec{z}_{T}\right)} \\
& \times\langle 0| \bar{q}(0) W_{u}^{\dagger}(+\infty, 0) h_{-} \gamma_{5} \text { [tr. link] } W_{u}(+\infty, z) q(z)\left|\pi^{+}(p)\right\rangle \\
& \times \sqrt{\frac{S\left(z_{T} ; y_{1}, y_{2}\right)}{S\left(z_{T} ; y_{1}, y_{u}\right) S\left(z_{T} ; y_{2}, y_{u}\right)}},
\end{aligned}
$$

where the soft function is defined as

$$
S\left(z_{T} ; y_{A}, y_{B}\right)=\frac{1}{N_{c}}\left\langle 0\left|W_{n_{B}}^{\dagger}\left(\infty, \vec{z}_{T}\right)_{c a} W_{n_{A}}\left(\infty, \vec{z}_{T}\right)_{a d} W_{n_{B}}(\infty, 0)_{b c} W_{n_{A}}^{\dagger}(\infty, 0)_{d b}\right| 0\right\rangle
$$

with $n_{A}$ and $n_{B}$ being the rapidities of the gauge-fixing vectors $n_{A}$ and $n_{B}$. This definition is unique, up to the choice of the ultraviolet renormalization prescription, provided that the following criteria are satisfied [20].

- The unsubtracted wave function only involves light-cone Wilson lines.

- Each soft factor has at most one off-light-cone Wilson line. 


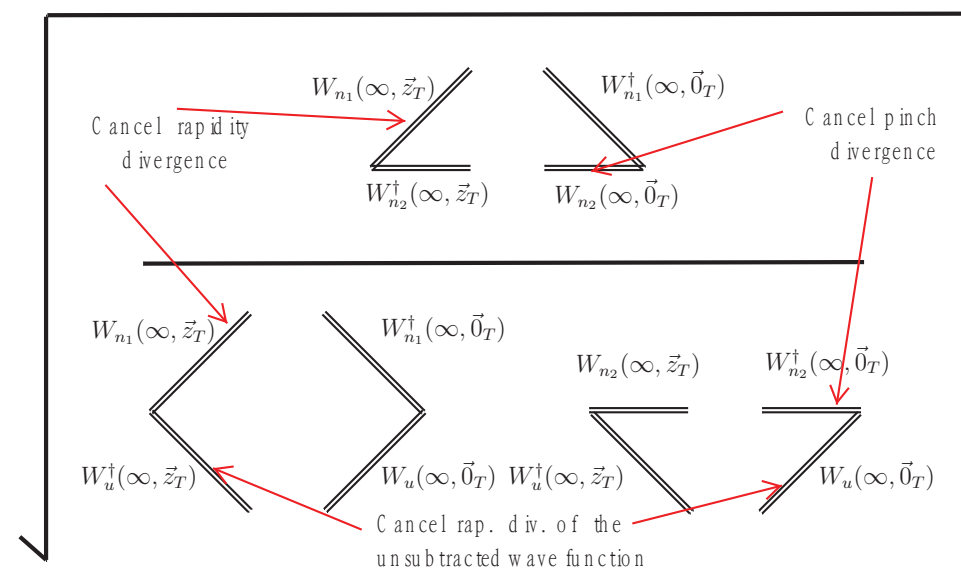

Figure 1. Diagrammatical representation of the soft subtraction in (10).

- The subtracted wave function is free of rapidity and pinch singularities and is defined as the product of the unsubtracted wave function and powers of the soft function in transverse coordinate space.

To demonstrate the cancellation mechanism in a transparent way, a detailed account of the rapidity and pinch structures of different pieces in the soft subtraction is display in figure 1 .

At this point, one might be curious to ask whether there exists an alternative definition of the TMD pion wave function with a simpler soft subtraction function. The key to answering this question consists in identifying the very origin of pinch singularities, which is due to the dipolar structure of the gauge links defining the TMD wave functions or parton densities. It is then a straightforward task to propose the following definition [19]

$$
\begin{aligned}
& \phi_{\pi}^{\text {new }}\left(x, \vec{k}_{T}, y_{2}, \mu\right)=\int \frac{d z_{-}}{2 \pi} \int \frac{d^{2} z_{T}}{(2 \pi)^{2}} e^{i\left(x p_{+} z_{-}-\vec{k}_{T} \cdot \vec{z}_{T}\right)}
\end{aligned}
$$

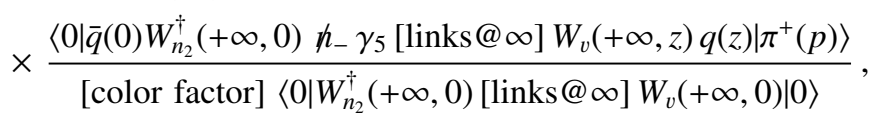

with two distinct off-light-cone gauge vectors $n_{2}$ and $v$. It is evident that the pinch singularity appeared in the naive definition (7) is now alleviated to a soft divergence in the unsubtracted wave function

$$
\phi_{\pi}^{\text {new }} \supset \int[d l] \frac{n_{2} \cdot v}{[l+i 0)]\left[n_{2} \cdot l+i 0\right][v \cdot l-i 0]} \delta\left(x^{\prime}-x+l_{+} / p_{+}\right) \delta^{(2)}\left(\vec{k}_{T}^{\prime}-\vec{k}_{T}+\vec{l}_{T}\right),
$$

which is further subtracted by the soft function with a rather simple gauge structure. The Wilson-line path defining the unsubtracted wave function is presented in figure 2 and a similar path of Wilson lines at infinity is used to define the soft function. Different choices for the gauge-link path may serve the same purpose, however, a more careful study is in demand to quantity the scheme dependence on the Wilson-line contour defining the TMD wave functions (see e.g., [21]).

The general definition (12) can be further reduced with some specific choices of two non-lightcone vectors $n_{2}$ and $v$. In particular, employing the orthogonal Wilson lines $\left(n_{2} \cdot v=0\right)$ no soft 


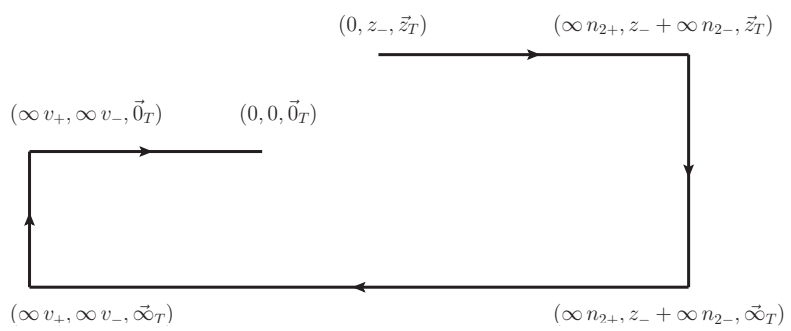

Figure 2. The Wilson-line path adopted to define the unsubtracted wave function (12).

subtraction will be needed in the TMD definition [19]

$$
\begin{aligned}
\phi_{\pi}^{\mathrm{I}}\left(x, \vec{k}_{T}, y_{2}, \mu\right)= & \int \frac{d z_{-}}{2 \pi} \int \frac{d^{2} z_{T}}{(2 \pi)^{2}} e^{i\left(x p_{+} z_{-}-\vec{k}_{T} \cdot \vec{z}_{T}\right)} \\
& \times\langle 0| \bar{q}(0) W_{n_{2}}^{\dagger}(+\infty, 0) h_{-} \gamma_{5}[\text { links@ } @] W_{v}(+\infty, z) q(z)\left|\pi^{+}(p)\right\rangle,
\end{aligned}
$$

with

$$
n_{2}=\left(e^{y_{2}}, e^{-y_{2}}, \overrightarrow{0}_{T}\right), \quad v=\left(-e^{y_{2}}, e^{-y_{2}}, \overrightarrow{0}_{T}\right) .
$$

One can readily verify that both of the two definitions (10) and (14) result in the same collinear logarithm for the infrared subtraction (without the contribution from the LSZ terms)

$$
\phi_{\pi}^{C, \mathrm{I}} \otimes H^{(0)}=-\frac{\alpha_{s} C_{F}}{4 \pi}[2 \ln x+3] \ln \left(\frac{k_{T}^{2}}{Q^{2}}\right) H^{(0)}\left(x, k_{T}\right)+(\text { IR finite terms }),
$$

at one-loop approximation, which reproduces the collinear behaviour of the one-loop QCD amplitude for the partonic Green function corresponding to $\gamma^{*} \rightarrow \pi \gamma$ at leading Fock-state approximation. Alternatively, one can facilitate the TMD definition (12) with the antiparallel Wilson lines

$$
\begin{aligned}
\phi_{\pi}^{\mathrm{II}}\left(x, \vec{k}_{T}, y_{2}, \mu\right)= & \int \frac{d z_{-}}{2 \pi} \int \frac{d^{2} z_{T}}{(2 \pi)^{2}} e^{i\left(x p_{+} z_{-}-\vec{k}_{T} \cdot \vec{z}_{T}\right)} \\
& \times \frac{\langle 0| \bar{q}(0) W_{n_{2}}^{\dagger}(+\infty, 0) \not h_{-} \gamma_{5}[\text { links@ } @] W_{n_{2}}(-\infty, z) q(z)\left|\pi^{+}(p)\right\rangle}{[\text { color factor }]\langle 0| W_{n_{2}}^{\dagger}(+\infty, 0)[\text { links@ } @] W_{n_{2}}(-\infty, 0)|0\rangle},
\end{aligned}
$$

which can be also shown to reproduce the very collinear structure in (16) and I will not expand the discussion for this definition.

\section{Equivalence of TMD definitions}

Now I will demonstrate the equivalence of different TMD definitions to all orders of the strong coupling in light of the arguments inspired from the rapidity evolution equations. First, I will explain that both of the definitions (10) and (14) will recover the naive definition (5) in the limit of vanishing regulators. One can readily verify that switching off the regulator in (10), i.e., $y_{2} \rightarrow-\infty\left(\Rightarrow y_{2}=y_{u}\right)$ implies that

$$
\sqrt{\frac{S\left(z_{T} ; y_{1}, y_{2}\right)}{S\left(z_{T} ; y_{1}, y_{u}\right) S\left(z_{T} ; y_{2}, y_{u}\right)}} \rightarrow 1
$$




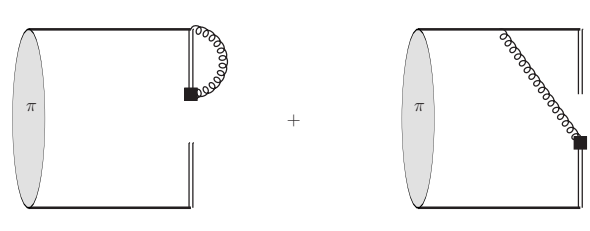

$\mathrm{K}$ function

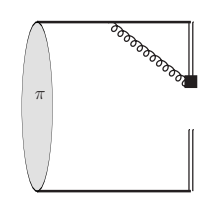

$\mathrm{G}$ function

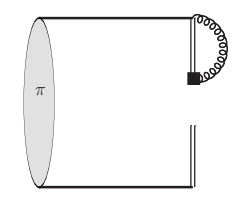

Figure 3. Graphic representation of the rapidity evolution kernels for the new TMD definition (14).

which evidently reduces the Collins' definition to (5). Similarly, setting $y_{2} \rightarrow-\infty\left(\Rightarrow n_{2}=v=n_{-}\right)$in (14) trivially gives rise to

$$
\phi_{\pi}^{\mathrm{I}}\left(x, \vec{k}_{T}, y_{2}, \mu\right) \rightarrow \phi_{\pi}^{\text {naive }}\left(x, \vec{k}_{T}, \mu\right) .
$$

We are then led to conclude that both Collins' and our proposals for the TMD definitions yield the same collinear divergence in the limit $y_{2}=y_{u} \rightarrow-\infty$. The remaining task is to show that the two definitions can bring about the same collinear behaviour for an arbitrary rapidity $y_{2}$. To this end, we need to examine the rapidity evolution equations for both definitions of the TMD pion wave function, employing the standard resummation techniques in QCD [1, 22].

Noticing that the rapidity dependence in Collins' definition (10) comes from the soft subtraction entirely and applying the routine chain rule for the rapidity derivative one can write

$$
\frac{d}{d y_{2}} \phi_{\pi}^{C}=\lim _{\substack{y_{1} \rightarrow+\infty \\ y_{u} \rightarrow-\infty}} \frac{1}{2}[\underbrace{\frac{S^{\prime}\left(z_{T} ; y_{1}, y_{2}\right)}{S\left(z_{T} ; y_{1}, y_{2}\right)}}_{K\left(z_{T} ; y_{1}, y_{2}\right)}-\underbrace{\frac{S^{\prime}\left(z_{T} ; y_{2}, y_{u}\right)}{S\left(z_{T} ; y_{2}, y_{u}\right)}}_{K\left(z_{T} ; y_{2}, y_{u}\right)}] \phi_{\pi}^{C} \approx \lim _{y_{1} \rightarrow+\infty} K\left(z_{T} ; y_{1}, y_{2}\right) \phi_{\pi}^{C},
$$

where the primed soft functions $S^{\prime}$ are computed from the Wilson-line diagrams defining the soft functions, with the Eikonal vertex $n_{2}^{\mu}$ replaced by a special vertex given by Eq. (4.4) in [19], and only the soft function $K\left(z_{T} ; y_{2}, y_{u}\right)$ enters the rapidity evolution of the TMD definition (10) in contrast to the original Collins-Soper-Sterman evolution [1,22].

Constructing the rapidity evolution for the newly proposed definition (14) is slightly more involved due to the appearance of two gauge vectors dependent on the same rapidity parameter $y_{2}$ and to the rapidity dependence of the unsubtracted wave function. It is nevertheless without the conceptual difficulty to derive the following evolution equation

$$
\frac{d}{d y_{2}} \phi_{\pi}^{\mathrm{I}}=\lim _{y_{1} \rightarrow+\infty}\left[K\left(z_{T} ; y_{1}, y_{2}\right)+G\left(y_{2}\right)\right] \phi_{\pi}^{\mathrm{I}}
$$

where the soft and hard functions can be computed from the effective diagrams presented in figure 3 . We can then readily conclude that the infrared behaviour of the TMD definition (14) is equivalent to that proposed by Collins (10), to all orders of the strong coupling, due to the infrared safety of the hard function $G\left(y_{2}\right)$.

\section{Concluding discussion}

To summarize, I presented a brief historic report on understanding the TMD factorization for hard exclusive processes, using the definition of TMD wave functions as an illustrative example. I provided a detailed account of justifying the new definition (12), with a simple soft function, applicable 
to a QCD description of $\gamma^{*} \rightarrow \pi \gamma$ at large momentum transfer. Furthermore, I demonstrated the equivalence of Collins' and our proposals for the TMD definitions in the infrared behaviours to all orders of perturbative expansion, with the aid of the rapidity evolution equations using the classical QCD resummation techniques. However, there remain many conceptual issues of constructing TMD factorization formulae for hard exclusive reactions as already emphasized above. Among these unsettled questions, the top priority should be to establish a definite power counting scheme for all the energy scales involved in a given problem, including the intrinsic transverse momentum. I therefore anticipate exciting developments of putting TMD factorization for hard exclusive processes on a solid ground, in addition to its phenomenological success.

\section{Acknowledgements}

I am grateful to Hsiang-nan Li for a very fruitful collaboration and to the organizers of POETIC6 for the generous finical support.

\section{References}

[1] J. Botts and G. F. Sterman, Nucl. Phys. B 325, 62 (1989).

[2] H. n. Li and G. F. Sterman, Nucl. Phys. B 381, 129 (1992).

[3] G. P. Lepage and S. J. Brodsky, Phys. Rev. D 22, 2157 (1980).

[4] J. C. Collins, Acta Phys. Polon. B 34, 3103 (2003) [hep-ph/0304122].

[5] S. Nandi and H. n. Li, Phys. Rev. D 76, 034008 (2007) [arXiv:0704.3790 [hep-ph]].

[6] F. Feng, J. P. Ma and Q. Wang, Phys. Lett. B 674, 176 (2009) [arXiv:0807.0296 [hep-ph]].

[7] H. n. Li, Y. L. Shen, Y. M. Wang and H. Zou, Phys. Rev. D 83, 054029 (2011) [arXiv:1012.4098 [hep-ph]].

[8] H. n. Li, Y. L. Shen and Y. M. Wang, Phys. Rev. D 85, 074004 (2012) [arXiv:1201.5066 [hep-ph]].

[9] W. F. Wang and Z. J. Xiao, Phys. Rev. D 86, 114025 (2012) [arXiv:1207.0265 [hep-ph]].

[10] H. N. Li, Y. L. Shen and Y. M. Wang, JHEP 1302, 008 (2013) [arXiv:1210.2978 [hep-ph]].

[11] H. N. Li, Y. L. Shen and Y. M. Wang, JHEP 1401, 004 (2014) [arXiv:1310.3672 [hep-ph]].

[12] S. Cheng, Y. Y. Fan, X. Yu, C. D. Lü and Z. J. Xiao, Phys. Rev. D 89, 094004 (2014) [arXiv:1402.5501 [hep-ph]].

[13] Y. L. Shen and Y. M. Wang, EPJ Web Conf. 80, 00047 (2014) [arXiv:1409.1048 [hep-ph]].

[14] Y. M. Wang, Int. J. Mod. Phys. Conf. Ser. 37, 1560049 (2015).

[15] S. S. Agaev, V. M. Braun, N. Offen and F. A. Porkert, Phys. Rev. D 83, 054020 (2011) [arXiv:1012.4671 [hep-ph]].

[16] J. Collins, "Foundations of perturbative QCD," Cambridge monographs on particle physics, nuclear physics and cosmology, 32.

[17] B. Melic, D. Mueller and K. Passek-Kumericki, Phys. Rev. D 68, 014013 (2003) [hep$\mathrm{ph} / 0212346]$.

[18] A. Bacchetta, D. Boer, M. Diehl and P. J. Mulders, JHEP 0808, 023 (2008) [arXiv:0803.0227 [hep-ph]].

[19] H. n. Li and Y. M. Wang, JHEP 1506, 013 (2015) [arXiv:1410.7274 [hep-ph]].

[20] J. Collins, Int. J. Mod. Phys. Conf. Ser. 4, 85 (2011) [arXiv:1107.4123 [hep-ph]].

[21] I. O. Cherednikov, T. Mertens and F. F. Van der Veken, "Wilson lines in quantum field theory,"

De Gruyter Stud. Math. Phys. 24 (2014).

[22] J. C. Collins and D. E. Soper, Nucl. Phys. B 193, 381 (1981) [Nucl. Phys. B 213, 545 (1983)]. 